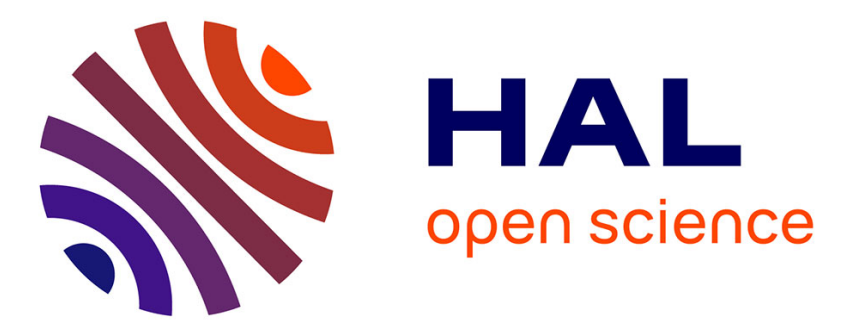

\title{
Slumping of non-Boussinesq density currents of various initial fractional depths: a comparison between direct numerical simulations and a recent shallow-water model
}

Thomas Bonometti, S. Balachandar

\section{- To cite this version:}

Thomas Bonometti, S. Balachandar. Slumping of non-Boussinesq density currents of various initial fractional depths: a comparison between direct numerical simulations and a recent shallow-water model. Computers and Fluids, 2010, vol. 39, pp. 729-734. 10.1016/j.compfluid.2009.11.008 . hal00862620

\section{HAL Id: hal-00862620 \\ https://hal.science/hal-00862620}

Submitted on 17 Sep 2013

HAL is a multi-disciplinary open access archive for the deposit and dissemination of scientific research documents, whether they are published or not. The documents may come from teaching and research institutions in France or abroad, or from public or private research centers.
L'archive ouverte pluridisciplinaire HAL, est destinée au dépôt et à la diffusion de documents scientifiques de niveau recherche, publiés ou non, émanant des établissements d'enseignement et de recherche français ou étrangers, des laboratoires publics ou privés. 


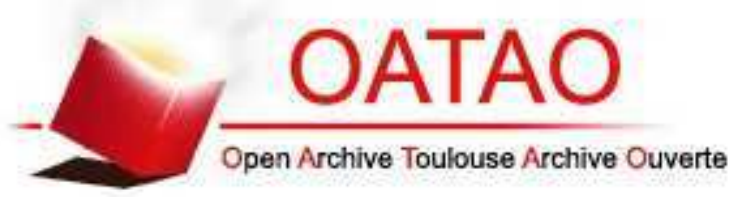

\section{Open Archive TOULOUSE Archive Ouverte (OATAO)}

OATAO is an open access repository that collects the work of Toulouse researchers and makes it freely available over the web where possible.

This is an author-deposited version published in : http://oatao.univ-toulouse.fr/ Eprints ID : 9410

To link to this article : DOI:10.1016/j.compfluid.2009.11.008

URL : http://dx.doi.org/10.1016/j.compfluid.2009.11.008

To cite this version : Bonometti, Thomas and Balachandar, S. Slumping of non-Boussinesq density currents of various initial fractional depths: a comparison between direct numerical simulations and a recent shallowwater model. (2010). Computers and Fluids, vol. 39 ( $\left.\mathrm{n}^{\circ} 4\right)$. pp. 729-734. ISSN 0045-7930

Any correspondance concerning this service should be sent to the repository administrator: staff-oatao@ listes-diff.inp-toulouse.fr 


\title{
Slumping of non-Boussinesq density currents of various initial fractional depths: A comparison between direct numerical simulations and a recent shallow-water model
}

\author{
Thomas Bonometti ${ }^{\mathrm{a}, \mathrm{b}, *}, \mathrm{~S}$. Balachandar ${ }^{\mathrm{c}}$ \\ a Université de Toulouse, INPT, UPS, IMFT - Institut de Mécanique des Fluides de Toulouse, Allée Camille Soula, F-31400 Toulouse, France \\ ${ }^{\mathrm{b}} \mathrm{CNRS}, \mathrm{IMFT}, \mathrm{F}-31400$ Toulouse, France \\ ${ }^{\mathrm{C}}$ Department of Mechanical and Aerospace Engineering, University of Florida, Gainesville, FL 32611, USA
}

Keywords:

Gravity currents

Non-Boussinesq current

Shallow-water model

Direct numerical simulations

\begin{abstract}
A B S T R A C T
Simulations of non-Boussinesq density currents of various initial fractional depths are performed, and a detailed comparison with a recent shallow-water model [18] is done for a wide range of density ratio $10^{-4} \leqslant \rho_{c} / \rho_{a} \leqslant 10^{4}$ and initial depth ratio $1 \leqslant H^{*} \leqslant 50$ in the regime of constant-speed initial phase. Good agreement is observed in a large region of the parameter space. The larger discrepancy is observed in the range of high $H^{*}$ and low- $\rho_{c} / \rho_{a}$ for which the shallow-water model overpredicts the velocity of the current. Two possible reasons are suspected, namely the fluid motion in the ambient fluid which is not accounted for in the model, and the choice of the model for the front condition.
\end{abstract}

\section{Introduction}

The dynamics of Boussinesq planar density currents of arbitrary initial depth ratio has been studied extensively because of their importance in various industrial and environmental problems. Laboratory experiments $[13,15]$, numerical investigations $[6,9,14]$ and analytical modeling $[1,10,11,15-17]$ have provided detailed information about the distance of propagation, the shape of the interface and the velocity regimes. The study of density currents of arbitrary density ratio is more complicated and most of the previous reported work has been restricted to the lock-exchange configuration $[2,4,7,12]$ for which the initial height of the current fluid is the same as that of the ambient (the only reported work of non-Boussinesq current for various initial depth ratio is Ref. [8]).

Recently Ungarish [J. Fluid Mech. 579, 373 (2007); Ref. [18] in the present paper] used a one-layer shallow-water model (see e.g. Refs. $[15,11]$ for Boussinesq flows) for the prediction of the shape and propagation of high Reynolds number density currents for the complete range of density ratio and initial depth ratio. This

\footnotetext{
* Corresponding author. Address: Université de Toulouse, INPT, UPS, IMFT Institut de Mécanique des Fluides de Toulouse, Allée Camille Soula, F-31400 Toulouse, France. Tel.: +33 5612859 52; fax: +33 561285990 .

E-mail addresses: thomas.bonometti@imft.fr (T. Bonometti), bala1s@ufl.edu (S. Balachandar).
}

model provides a useful tool for understanding and modeling density current dynamics, since Boussinesq and non-Boussinesq currents are treated in a unified manner. Among other results, the model gives the height $h_{N}$ and the velocity $u_{N}$ of the current during the constant-speed initial phase (note that the paper also predicted the self-similar propagation for later times). This information is of critical interest in hazardous situations such as fires in tunnels, dam break or accidental release of toxic gases or liquids. However, as emphasized by Ungarish [18], the model's validity is largely untested and "given the paucity of the available data, the main deficiency of the model is the unknown practical limit of applicability".

The objective of this paper is to fill this gap using direct numerical simulations. We consider the propagation of a density current of density $\rho_{c}$ and initial depth $h_{0}$ into an ambient fluid of density $\rho_{a}$ and initial depth $H$ (Fig. 1). Depending on the sign of $\rho_{a}-\rho_{c}$, we refer to a bottom density current $\left(\rho_{a}-\rho_{c}<0\right)$ or a surface density current $\left(\rho_{a}-\rho_{c}>0\right)$. The current is released from a lock of initial position $x_{0}$ and height $h_{0}$. This configuration is strictly the same as in Ref. [18]. In this paper we report results of a series of twodimensional simulations of planar density currents for a wide range of density ratios $10^{-4} \leqslant \rho_{c} / \rho_{a} \leqslant 10^{4}$ and initial depth ratios $1 \leqslant H^{*} \leqslant 50$ (where $H^{*}=H / h_{0}$ ). These simulations allow us to verify the strengths and limitations of the shallow-water model developed in Ref. [18] in accurately predicting current height and velocity over a wide range of density and initial depth ratios. 


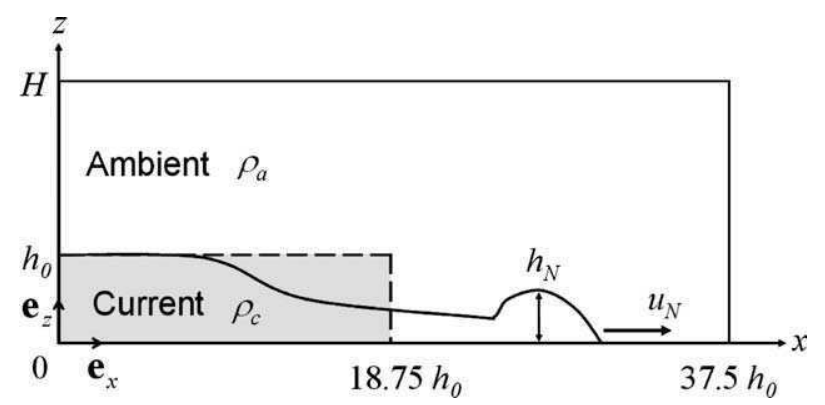

Fig. 1. The physical configuration and nomenclature used in this work. The dashed line represents the initial separation between fluids. The solid line represents the interface at later times. The gravity vector is defined as $\mathbf{g}=g\left(\rho_{a}-\rho_{c}\right) /\left|\rho_{a}-\rho_{c}\right| \boldsymbol{e}_{z}$.

\section{Shallow-water and Navier-Stokes equations}

\subsection{Shallow-water model}

In Ref. [18], Ungarish used a one-layer shallow-water theory for the prediction of the shape and propagation of high-Re density currents for the complete range of density ratio $\rho_{c} / \rho_{a}$ and initial depth ratio $H^{*}$. In the following, we briefly describe the assumptions and equations used in the model to obtain the height $h_{N}$ and the velocity $u_{N}$ of the current during the constant-speed initial phase.

The classical assumptions of the shallow-water analysis are (i) the fluids are incompressible, immiscible and inviscid and (ii) the current is shallow, that is $h_{0} / x_{0}<<1$. From these assumptions it follows that the pressure is hydrostatic. To close the system of equations an additional boundary condition is specified at the front of the current located at $x_{N}$ (see Refs. $[15,18]$ ). Ungarish's model [18] makes use of the condition

$u_{N}=\left(g h_{N}|\Delta \rho| / \rho_{a}\right)^{1 / 2} \mathrm{Fr}$,

where the Froude number, $F r$, is a function of the depth ratio $h_{N} / H$ and is taken to be the Benjamin's relation given by

$\operatorname{Fr}\left(h_{N} / H\right)=\left[\frac{\left(2-h_{N} / H\right)\left(1-h_{N} / H\right)}{\left(1+h_{N} / H\right)}\right]^{1 / 2}$.

Further noting that during the slumping stage, the domain of fluid trailing the nose is a rectangle of constant height, and using the fact that the energy in the domain cannot increase, Ungarish matched the shallow-water solution of the forward-propagating characteristic with Benjamin's front condition to obtain the height and velocity of the fronts. The height of the current is given by the following implicit equation

$2 \sqrt{\frac{\rho_{a}}{\rho_{c}}}\left(1-\sqrt{\frac{h_{N}}{h_{0}}}\right)=\sqrt{\frac{h_{N}}{h_{0}}} \operatorname{Fr}\left(h_{N} / H\right)$.

where the Froude number relation is given in (2). If we then apply the additional energy constraint, the maximum height of the current can be limited to $H / 2$ and we obtain $h_{N}=\min \left(h_{N}, H / 2\right)$. The velocity of the current can then be determined from (1). More details can be found in Refs. $[18,19]$.

\subsection{Navier-Stokes equations and its numerical treatment}

Assuming the current and the ambient fluid to be Newtonian, immiscible and incompressible, the evolution of the flow is described using the one-fluid formulation of the Navier-Stokes equations, namely

$\rho \frac{\partial \mathbf{u}}{\partial t}+\rho \mathbf{u} \cdot \nabla \mathbf{u}=-\nabla p+\rho \mathbf{g}+\nabla \cdot\left[\mu\left(\nabla \mathbf{u}+{ }^{t} \nabla \mathbf{u}\right)\right] ; \quad \nabla \cdot \mathbf{u}=0$. where $\boldsymbol{u}, p, \rho$ and $\mu$ are the local velocity, pressure, density and dynamic viscosity in the flow, respectively, and $\boldsymbol{g}$ denotes gravity. The local volume fraction of the current fluid obeys

$\frac{\partial C}{\partial t}+\mathbf{u} \cdot \nabla C=0$

This volume fraction equals one (resp. zero) in cells filled with the current fluid (resp. ambient) and takes intermediate values in cells belonging to the transition region. The local density and dynamic viscosity are evaluated using a linear interpolation, namely

$\rho=C \rho_{c}+(1-C) \rho_{a} ; \quad \mu=C \mu_{c}+(1-C) \mu_{a}$.

Briefly, the numerical technique is a finite-volume front-capturing method with no interface reconstruction that allows simulation of non-diffusive fluids of arbitrary density ratio. Momentum equations are solved on a staggered grid using second-order centered differences for the spatial discretization and a third-order Runge-Kutta/Crank-Nicolson method for the temporal discretization. The transport equation of the volume fraction (5) is solved using a modified Zalesak scheme [20]. We refer the reader to Refs. [4,5] for more details on the numerical algorithm and solution methods used here.

In this approach, the evolution equation of the volume fraction is hyperbolic. This is equivalent to choosing a Schmidt number $S c \rightarrow \infty$ (the Schmidt number being defined as the ratio of the kinematic viscosity to the molecular diffusivity). Note that although no physical diffusivity is introduced in (5), the numerical thickness of the interface is not strictly zero as it is typically resolved over three grid cells [5]. Therefore a finite effective Schmidt number can be estimated, which depends somewhat on $R e$ and on the degree of spatial resolution. Based on extensive tests of measurement of the interface thickness, Bonometti and Balachandar (Ref. [3]) estimated the effective Schmidt number to be of $O\left(10^{3}\right)$.

In the following, we assume the same constant dynamical viscosity for both fluids, that is $\mu_{c}=\mu_{a}$. Note that the present numerical methodology allows for simulations of arbitrary viscosity ratios. As shown in Ref. [4] (see their Appendix B), provided the Reynolds number, based on the heavier fluid, is large (i.e. $R e \geqslant O\left(10^{3}\right)$ ), the evolution of density current is mostly independent of the choice of viscosity ratio. In particular, at high Reynolds numbers quantities such as the front velocity to be discussed here are quite similar irrespective of whether constant kinematic or constant dynamic viscosity is employed. We have performed additional tests (not shown here) with different initial fractional depths $\mathrm{H}^{*}$ and they revealed that the near-independence of results to viscosity ratio at high Reynolds numbers holds even when $H^{*}$ is not unity. Thus the results and conclusions to be presented are reflective of what can be expected in actual gravity currents of immiscible fluids of arbitrary density and viscosity ratios.

Simulations are performed for flows at a Reynolds number $R e=\sqrt{g^{\prime} h_{0}} h_{0} / v$ of $2.5 \times 10^{4}$ (here $g^{\prime}=g\left|\rho_{a}-\rho_{c}\right| / \max \left(\rho_{a}, \rho_{c}\right)$, and $v$ is the kinematic viscosity of the heavy fluid). Preliminary tests were performed for two other Reynolds numbers, namely $R e=1.25 \times 10^{4}$ and $5 \times 10^{4}$. As shown in Table 1 , these tests revealed almost no difference (i.e. less than $1 \%$ ) in both the front velocity and the height of the current. In addition, we recorded the temporal evolution of the

Table 1

Front velocity $u_{N}$ and current height $h_{N}$ for various Reynolds numbers $\left(\rho_{c} / \rho_{a}=10^{-3}\right.$ and $H^{*}=50$ ).

\begin{tabular}{lll}
\hline$R e$ & $u_{N} /\left(g^{\prime} h_{0}\right)^{1 / 2}$ & $h_{N} / h_{0}$ \\
\hline $1.25 \times 10^{4}$ & $1.098 \pm 0.011$ & 1.451 \\
$2.5 \times 10^{4}$ & $1.103 \pm 0.012$ & 1.456 \\
$5 \times 10^{4}$ & $1.105 \pm 0.011$ & 1.459 \\
\hline
\end{tabular}


overall mechanical energy. In all cases the relative variation of the total energy remains negligibly small during the entire duration of the simulation (less than $0.01 \%$ ).

We estimated the minimum value of the ratio $x_{0} / h_{0}$ (initial length to initial height of the current) that would allow the current to remain in the slumping phase as it travels forward for about $20 h_{0}$ (Fig. 1). The choice of $x_{0} / h_{0} \approx 20$ was computationally observed to be sufficient to maintain the slumping phase over the entire duration of simulation in all cases considered. Therefore, with the present configuration, the computed front velocity is expected not to be affected by the presence of the lateral walls.

The simulations are two-dimensional and are performed within a rectangular $(x, z)$ domain of size $37.5 h_{0} \times H$. We use a $2400 \times 300$ grid with a uniform spacing in the $x$-direction $\left(\Delta x / h_{0}=1 / 64\right)$. In the $z$-direction, a uniform spacing of $\Delta z / h_{0}=6.25 \times 10^{-3}$, corresponding to 160 grid cells per initial current height, is used over the region $0 \leqslant z \leqslant 1.5 h_{0}$ (this region covers the density current and a significant portion of the ambient fluid entrained by the current), while larger cells are used above this region, following an arithmetic progression. Free-slip boundary conditions for the velocity (unless otherwise specified) and zero normal gradient for the volume fraction are imposed on the top, bottom and lateral boundaries. Computations are stopped before the density current comes close to the right vertical boundary, to avoid contamination of the results by confinement effects. In the specific case $H^{*}=1$, a smaller grid of $2400 \times 160$ cells was used (same spacing as mentioned above). Note that the present resolution is similar to that used in Ref. [4].

\section{Results and discussion}

In all the results presented in this paper, the computation starts from rest, the dynamical viscosity ratio and the Reynolds number are set to 1 and $2.5 \times 10^{4}$, respectively, while we vary the density ratio and the initial depth ratio. Note that in order to keep the Reynolds number constant while the density ratio varies, we modify the viscosity of the fluids accordingly. For each simulation, we record the temporal evolution of the front position $x_{N}$ defined as the maximum value of $x$ for which the equivalent height $\bar{h}$ is non-zero, where $\bar{h}$ is defined as [6,13]:

$\bar{h}(x, t)=\int_{0}^{H}\left(\rho(x, z)-\rho_{a}\right) /\left(\rho_{c}-\rho_{a}\right) d z$

Here $\rho$ is the local value of the density field. A sample of one thousand points was taken from the temporal evolution of the front position (over the range $5 \leqslant t \sqrt{g^{\prime} / h_{0}} \leqslant 10$ ), and used to compute the mean front velocity $u_{N}$. Additionally, we define the height of the current $h_{N}$ as the maximum value of $\bar{h}$ in the region close to the current front (typically of width $3 h_{0}$ behind the front). The measurement of $h_{N}$ at different time instances revealed that $h_{N}$ remains roughly constant during the constant-speed initial phase.

\subsection{Front velocity}

In Fig. 2, the front velocity (symbols) is compared with Ungarish's shallow-water model [18] (lines), for density currents of various density ratios and two different initial depth ratios, namely $H^{*}=1$ and 50 , respectively. Note that intermediate initial depth ratios were also investigated and the results were in essential agreement and therefore for clarity they are not presented here. The agreement is fairly good in the case $H^{*}=1$ (lock-exchange configuration) for the complete range of density ratio shown here. Indeed, the front velocity of surface density currents $\left(10^{-4} \leqslant \rho_{c} / \rho_{a} \leqslant 1\right)$ predicted by the shallow-water model agrees within $1 \%$ with the simulations while for bottom density currents $\left(1 \leqslant \rho_{c} / \rho_{a} \leqslant 10^{4}\right)$ the maximum discrepancy is less than $10 \%$. Such discrepancy

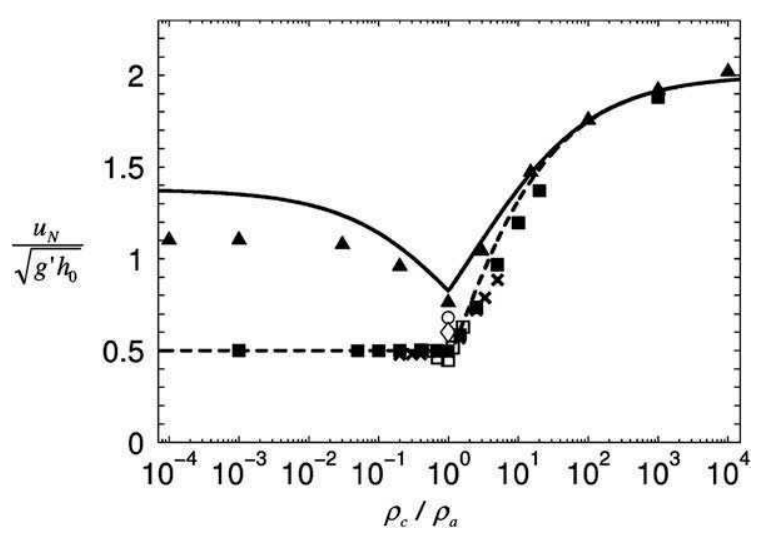

Fig. 2. Front velocity as a function of the density ratio. The solid (resp. dashed) line is the prediction of Ungarish's shallow-water model [18] for $H^{*}=50\left(\right.$ resp. $\left.H^{*}=1\right)$. Triangles (resp. solid squares) are computational results for $H^{*}=50\left(\right.$ resp. $\left.H^{*}=1\right)$. Crosses are numerical results of Ref. [2] $\left(H^{*}=1\right)$. Experimental results of Ref. [12] (open square: $H^{*}=1$ ) and Ref. [15] (diamond: $H^{*}=4$; circle: $H^{*} \approx 15$ ) are also plotted for comparison.

was previously observed in Ref. [4] for $10^{-1} \leqslant \rho_{c} / \rho_{a} \leqslant 6 \times 10^{-1}$, and was attributed to dissipation stemming from vortical structures generated near the front of the current.

For $H^{*}=50$ good agreement is observed for bottom density currents $\left(1 \leqslant \rho_{c} / \rho_{a} \leqslant 10^{4}\right)$. In line with the shallow-water model prediction, the results become independent of $H^{*}$ as the density ratio increases. For instance, the front velocities of the $H^{*}=1$ and $H^{*}=50$-currents are nearly identical for $\rho_{c} / \rho_{a}=10^{3}$. The situation is different for surface density currents. As shown in Fig. 2, we observe an increasing departure between the predicted (solid line) and computed velocities as the density ratio is decreased. The shallow-water model overestimates the front velocity by at most $25 \%$ for the smallest density ratios investigated here. We also plot for comparison the experimental results of Ref. [15] in the Boussinesq limit at intermediate initial fractional depths, namely $H^{*}=4$ and $H^{*} \approx 15$ (see the diamond and circle symbols). A monotonic increase in the velocity is observed from $H^{*}=1$ to $H^{*}=50$. Comparison between the experimental results and the present computational results is in general quite good.

\subsection{Front height}

The shape of Boussinesq density currents of arbitrary initial fractional depth has been extensively described in the literature $[6,9,10,13,15]$ as well as that of non-Boussinesq currents in the lock-exchange configuration $[2,4,7,8,12]$. However, the description of non-Boussinesq surface density currents in a deep ambient is much less documented. Therefore we plot in Fig. 3 the temporal evolution of the shape of a surface density current $\left(H^{*}=50\right.$ and $\rho_{c}\left(\rho_{a}=10^{-3}\right)$. Soon after the removal of the gate, the head of the current is clearly visible and takes the shape of a spherical cap. At the same time, a surface perturbation is formed behind the head. The distance separating the surface perturbation from the current head is observed to grow in time while the height of the current remains approximately constant in between. Therefore, for the times considered here, the shape of the surface density current can be decomposed into a head, a surface perturbation (note that some small vortical structures are discernable at the junction with the head) and a tail further downstream.

Fig. 4 shows the height of the current, $h_{N}$, for the complete range of density ratio and two initial depth ratios of $H^{*}=1$ and 50, respectively. In the case $H^{*}=1$, Ungarish's model predicts that the surface density current is choked to occupy only half of the 


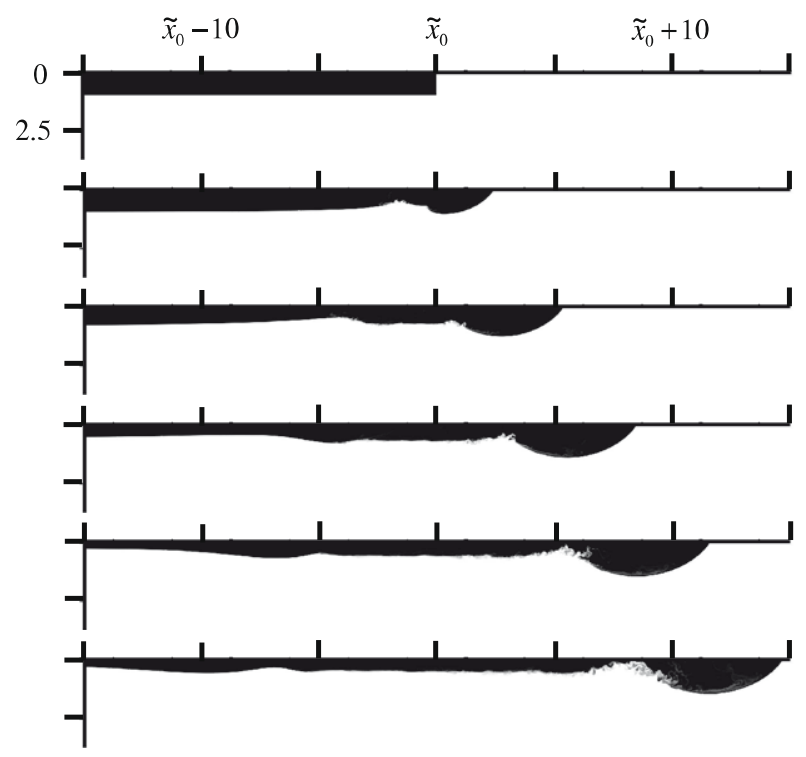

Fig. 3. Temporal evolution of the shape of a surface density current (shadowgraph) for $H^{*}=50$ and $\rho_{c} / \rho_{a}=10^{-3}$. Time interval between successive views is $\Delta t \sqrt{g^{\prime} / h_{0}}=2.827$. Axes are scaled by $h_{0}$.

height of the channel (i.e. $h_{N} / h_{0}=1 / 2$ ), while the bottom density current propagates mainly with unrestricted height, the latter being a decreasing function of the density ratio. These results are in full agreement with the present simulations and the agreement extends for all the density ratios investigated here.

For currents in a deep ambient $\left(H^{*}=50\right)$, good qualitative agreement is observed, but only partial quantitative agreement is found. Indeed both theoretical and computational results predict a smooth decrease of the height of the current with density ratio for $\rho_{c} / \rho_{a} \leqslant O\left(10^{-2}\right)$, followed by a steeper drop for $O\left(10^{-2}\right) \leqslant \rho_{c} /$ $\rho_{a} \leqslant O(10)$ and eventually a smoother decrease for $\rho_{c} / \rho_{a} \geqslant$ $O(10)$. In addition, quantitative agreement is found in the latter range of density ratio where the height of the current is only a small fraction of the initial depth ratio. In contrast, the height of the current is increasingly underestimated by the shallow-water theory as $\rho_{c} / \rho_{a}$ is decreased.

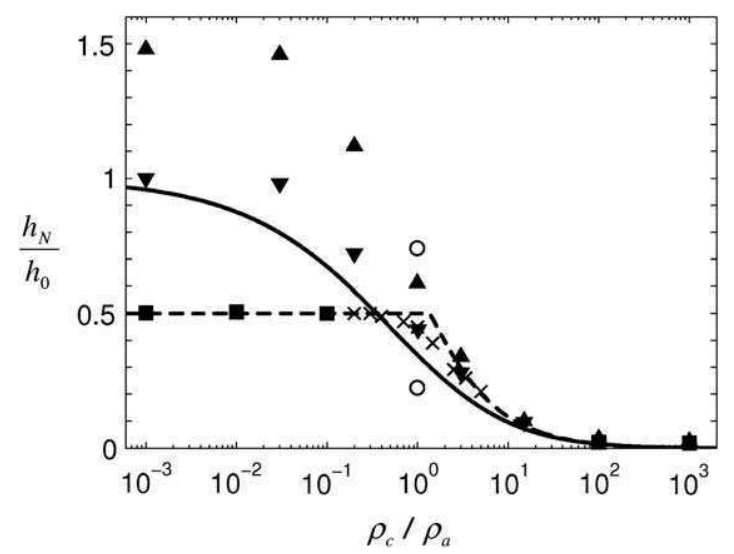

Fig. 4. Height of the head as a function of the density ratio. The solid (resp. dashed) line is the prediction of Ungarish's shallow-water model [18] for $H^{*}=50$ (resp. $H^{*}=1$ ). Upward triangles (resp. squares) are computational results for $H^{*}=50$ (resp. $H^{*}=1$ ). Downward triangles are the average height of the head of the current $\left(H^{*}=50\right)$. Crosses are numerical results of Ref. [2] $\left(H^{*}=1\right)$. Experimental results of Rottman and Simpson [15] are also plotted for comparison (case $H^{*}=4$ ). These correspond to the measured height including only the unmixed layer (lower circle) or both the mixed and unmixed layers (upper circle).
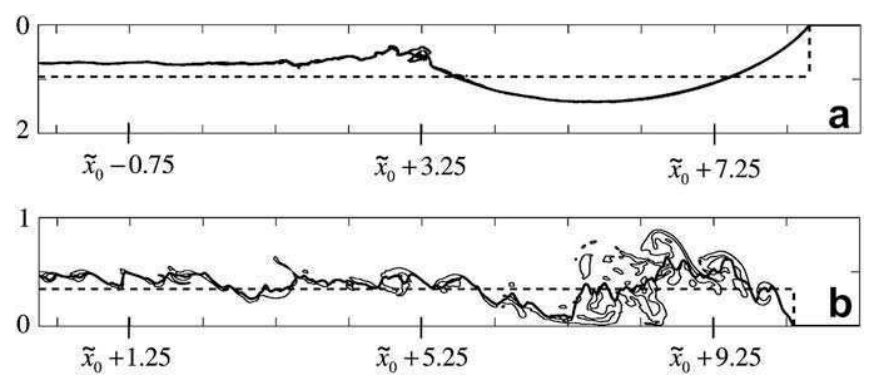

Fig. 5. Instantaneous shape of a surface density current and a Boussinesq density current, respectively. (a) $H^{*}=50 ; \rho_{c} / \rho_{a}=10^{-3} ; t \sqrt{g^{\prime} / h_{0}}=8.5$, (b) $H^{*}=50 ; \rho_{c} / \rho_{a}=$ $1.01 ; t \sqrt{g^{\prime} / h_{0}}=1.4$. The thick solid line is $\bar{h}$ as defined in (1). Thin dotted lines are contours of the iso-value $\left(\rho_{c}+\rho_{a}\right) / 2$. For comparison, a rectangle (dashed line) of dimension $x_{N} \times h_{N}$ is also plotted, the value of $h_{N}$ being that predicted by Ungarish's shallow-water model [18]. Axes are scaled by $h_{0}$.

Note that for large values of $H^{*}$ and $O(1)$ values of $\rho_{c} / \rho_{a}$, strong mixing in the vicinity of the current head was observed in the simulations (Fig. 5), in agreement with experimental observations [15]. We plot in Fig. 4 the experimental results of Rottman and Simpson [15] who measured the height of the current by either including the mixed layer (upper circle) or only the unmixed layer (lower circle). Note that in this case of Boussinesq limit, both the computed and theoretical values of $h_{N}$ fall in between the two experimental values. In contrast, for lower density ratios, the head of the surface density current observed in the simulations is clearly separated from the body of the current (see Figs. 3 and 5a), eliminating ambiguity about the definition and the measurement of the current height. However, previous investigations have indicated that the appropriate definition for the front depth is still an open question (see e.g. Refs. [11,13]). For instance, we plot in Fig. 4 the value of $\bar{h}$ averaged over the head of the current, i.e. between the minimum and the front (downward triangles). With this definition, the agreement with the model is greatly improved. In addition we plotted in Fig. 5 instantaneous shapes of a surface density current and a Boussinesq density current, respectively. The thick solid line is $\bar{h}$ as defined in (7) while the thin dotted lines are contours of the iso-value $\left(\rho_{c}+\rho_{a}\right) / 2$. For comparison, a rectangle of dimension $x_{N} \times h_{N}$ is also plotted, the value of $h_{N}$ being that predicted by Ungarish's shallow-water model [18]. Local differences between the predicted and computed heights are observable while the overall predicted and computed heights (say averaged along the streamwise direction) are in reasonable agreement. Clearly, the local differences are due to the presence of vortical structures observed along the body of the simulated currents, stemming from the interaction of the current with the ambient fluid, which are not taken into account in the analytical model. In any case, Figs. 4 and 5 indicate that caution must be exercised regarding the proper definition of the height of the front.

\subsection{Possible reasons for the observed differences}

In Ref. [18], Ungarish estimated that the effects of the ambient fluid on the dynamics of the density currents can be neglected provided $H^{*} \rho_{c} / \rho_{a}>2$ approximately. For $H^{*}=1$ this leads to the condition $\rho_{c} / \rho_{a}>2$. It can therefore be expected that the agreement between the simulations and the model breaks down for $\rho_{c} / \rho_{a}<2$. However, as was shown in Ref. [18], an additional energy constraint arises for $\rho_{c} / \rho_{a}<1.373$ (that is the flow is choked at half the initial depth). This sets the velocity of the current to be that imposed by the front condition, and as a result the agreement is excellent (see the comparison between the dashed line and the squares in Figs. 2 and 4). For $H^{*}=50$ the condition for neglecting the ambient fluid dynamics reduces to $\rho_{c} / \rho_{a}>4 \times 10^{-2}$. This is in 
reasonable agreement with our results for which the discrepancy is maximum for density ratios in the range $\rho_{c} / \rho_{a}<4 \times 10^{-2}$ approximately. Interestingly, the largest error in the speed of propagation is observed to be at most $25 \%$ for a density ratio that is 400 times smaller than the limit of validity, and the qualitative agreement is quite good. This robustness calls for some further theoretical and numerical investigation. Overall, we may conclude that one possible reason for the discrepancy between the shallow-water model and the computed velocities of surface density currents in deep ambient is the fact that the model neglects the fluid motion in the ambient fluid. Thus, under such conditions a two-layer model (see e.g. Ref. [19]), where both the motion of the current as well as the ambient is taken into account, can be expected to perform better.

As mentioned in Section 2.1, to obtain realistic solutions, the system of shallow-water equations must be subjected to a boundary condition at the front. Ungarish's model [18] makes use of Benjamin's Froude number function (2). Another well-known front condition often used in shallow-water models is the experimental correlation proposed by Huppert and Simpson (Ref. [10]) which reads:

$\operatorname{Fr}\left(h_{N} / H\right)= \begin{cases}1.19 & \left(0 \leqslant h_{N} / H \leqslant 0.075\right) \\ 0.5\left(h_{N} / H\right)^{-1 / 3} & \left(0.075 \leqslant h_{N} / H \leqslant 1\right)\end{cases}$

We plot in Fig. 6 the front velocity as a function of the density ratio for the front condition (8) used in conjunction with the one-layer shallow-water equations [Note that condition (2) corresponds to the original model of Ungarish [18], while we modified the latter to include condition (8) in Fig. 6]. With the use of the Huppert and Simspon's front condition, the agreement between the model and the simulations is significantly better (the maximum discrepancy is $9 \%$ ). Note that conditions (2) and (8) give comparable results for the prediction of the height of the front $h_{N}$ (Figs. 4 and 7). Clearly, the shallow-water model is quite sensitive to the choice of the front condition in the range of high $H^{*}$-low $\rho_{c} / \rho_{a}$. Therefore, a two-layer shallow-water theory is desirable to disentangle the possible conjugate effects of the fluid motion in the ambient fluid from that of the front condition in this range of parameters.

It was recently shown in Ref. [4] that, in the lock-exchange configuration $\left(H^{*}=1\right)$, wall friction could significantly reduce the speed of bottom density currents, especially in the approximate range $\rho_{c} / \rho_{a}>5$. In order to assess the relevance of this result to density currents of arbitrary initial fractional depth, we performed

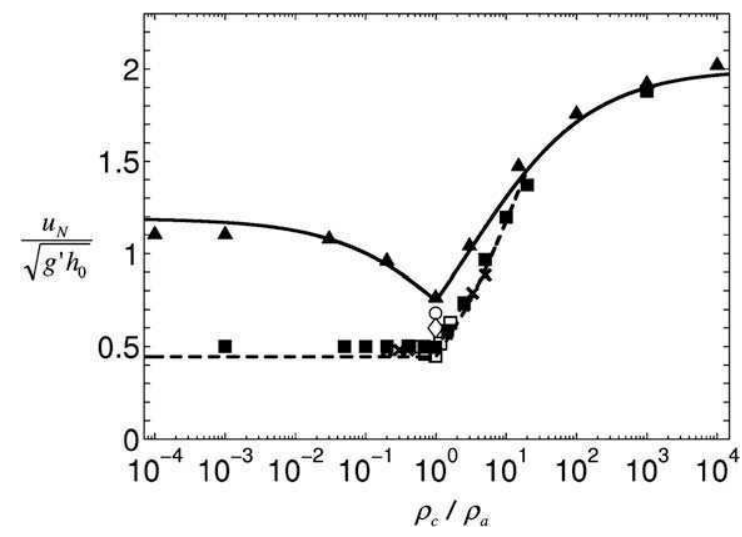

Fig. 6. Front velocity as a function of the density ratio. The solid (resp. dashed) line is the prediction of Ungarish's modified model (i.e. with Huppert \& Simpson's front condition (8)) for $H^{*}=50$ (resp. $H^{*}=1$ ). Triangles (resp. solid squares) are computational results for $H^{*}=50$ (resp. $H^{*}=1$ ). Crosses are numerical results of Ref. [2] $\left(H^{*}=1\right)$. Experimental results of Ref. [12] (open square: $H^{*}=1$ ) and Ref. [15] (diamond: $H^{*}=4$; circle: $H^{*} \approx 15$ ) are also plotted for comparison.

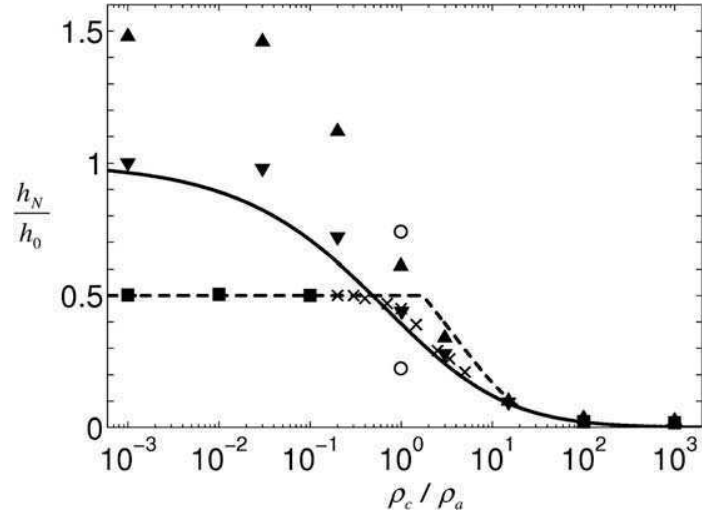

Fig. 7. Height of the head as a function of the density ratio. The solid (resp. dashed) line is the prediction of Ungarish's modified model (i.e. with Huppert \& Simpson's front condition (8)) for $H^{*}=50$ (resp. $H^{*}=1$ ). Upward triangles (resp. squares) are computational results for $H^{*}=50\left(\right.$ resp. $\left.H^{*}=1\right)$. Downward triangles are the average height of the head of the current $\left(H^{*}=50\right)$. Crosses are numerical results of Ref. [2] $\left(H^{*}=1\right)$. Experimental results of Rottman and Simpson [15] are also plotted for comparison (case $H^{*}=4$ ). These correspond to the measured height including only the unmixed layer (lower circle) or both the mixed and unmixed layers (upper circle)

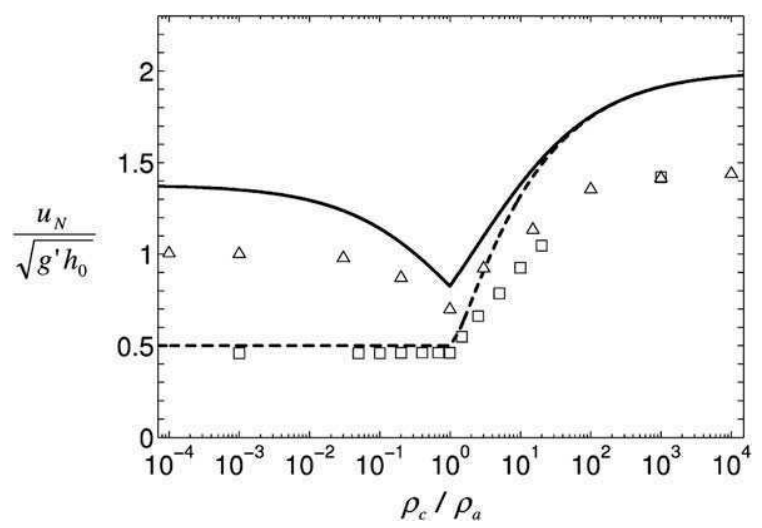

Fig. 8. Front velocity as a function of the density ratio. The solid (resp. dashed) line is the prediction of Ungarish's original shallow-water model [18] for $H^{*}=50$ (resp. $H^{*}=1$ ). Triangles (resp. squares) are computational results for $H^{*}=50$ (resp. $H^{*}=1$ ) with the use of a no-slip condition at the boundary on which the current spreads.

the same simulations as those in Fig. 2 with no-slip boundary conditions. Results are displayed in Fig. 8. The comparison between Figs. 2 and 8 suggests that the impact of the wall friction is significant for bottom density currents of arbitrary $H^{*}$ for density ratios larger than about 5 . Below this value the velocity difference between the free-slip and the no-slip cases is less than $10 \%$. As for the height of the currents, a small increase is observed but the variation is found to be less than a few percent for the complete range of $\rho_{c} / \rho_{a}$ and $H^{*}$. Overall, Fig. 8 extends the observations of Ref. [4] for lock-exchange flows to currents of arbitrary initial depth ratio that wall effects modify the velocity of propagation of highly contrasted bottom density currents.

\section{Summary}

Two-dimensional simulations of non-Boussinesq density currents of various initial fractional depths were performed, to provide a detailed comparison with a recent shallow-water model (Ref. [18]). Good agreement was observed in a wide range of initial fractional depth and density ratios. The larger discrepancy was observed in the range of high $H^{*}$ and low $\rho_{c} / \rho_{a}$ for which the original model overpredicts (resp. underpredicts) the velocity (resp. height) 
of the current during the constant-speed initial phase. Two possible reasons are suspected, namely the fluid motion in the ambient fluid which is not accounted for in the model, and the choice of the model for the front condition. Two-layer shallow-water models for arbitrary initial fractional depth and density ratio are therefore desirable to clearly identify the contribution of each effect.

This work focused on the initial slumping phase of non-Boussinesq density currents of various initial fractional depths. It could be extended in many ways. First, the analysis of the self-similar inertial and viscous regimes that occur immediately after the slumping phase is required to test the accuracy of the inviscid shallow-water models for the complete evolution of the density currents. It is also of interest to explore the effect of the threedimensionalities of the flow on the dynamics of non-Boussinesq density currents, as done for instance by Ref. [6] in the Boussinesq limit. The computational cost to achieve such three-dimensional simulations is quite large at the present time so that this is beyond the scope of the present paper. Nevertheless these directions are the next steps of our work.

\section{Acknowledgments}

We wish to thank M. Ungarish for drawing our attention on the possible effect of the front condition on the shallow-water results for surface density currents in deep ambient. We are grateful to J. Magnaudet for the use of the JADIM code. This work benefited from the NSF Grant EAR-0609712.

\section{References}

[1] Benjamin TB. Density currents and related phenomena. J Fluid Mech $1968 ; 31: 209-48$.
[2] Birman V, Martin JE, Meiburg E. The non-Boussinesq lock-exchange problem. Part 2. High-resolution simulations. J Fluid Mech 2005;537:125-44.

[3] Bonometti T, Balachandar S. Effect of Schmidt number on the structure and propagation of density currents. Theor Comput Fluid Dyn 2008;22:341-61.

[4] Bonometti T, Balachandar S, Magnaudet J. Wall effects in non-Boussinesq density currents. J Fluid Mech 2008;616:445-75.

[5] Bonometti T, Magnaudet J. An interface-capturing method for incompressible two-phase flows. Validation and application to bubble dynamics. Int J Multiphase Flow 2007;33:109-33.

[6] Cantero MI, Lee JR, Balachandar S, Garcia MH. On the front velocity of gravity currents. J Fluid Mech 2007:586:1-39.

[7] Etienne J, Hopfinger EJ, Saramito P. Numerical simulations of high density ratio lock-exchange flows. Phys Fluids 2005;17:036601.

[8] Gröbelbauer HP, Fanneløp TK, Britter RE. The propagation of intrusion fronts of high density ratio. J Fluid Mech 1993;250:669-87.

[9] Härtel C, Meiburg E, Necker F. Analysis and direct numerical simulation of the flow at a gravity-current head. Part 1. Flow topology and front speed for slip and no-slip boundaries. J Fluid Mech 2000;418:189-212.

[10] Huppert H, Simpson J. The slumping of gravity currents. J Fluid Mech 1980;99:785-99.

[11] Klemp JB, Rotunno R, Skamarock WC. On the dynamics of density currents in a channel. J Fluid Mech 1994;269:169-98.

[12] Lowe RJ, Rottman JW, Linden PF. The non-Boussinesq lock-exchange problem. Part 1. Theory and experiments. J Fluid Mech 2005;537:101-24.

[13] Marino B, Thomas L, Linden P. The front condition for density currents. J Fluid Mech 2005;536:49-78.

[14] Ozgökmen T, Fischer P, Duan J, Iliescu T. Three-dimensional turbulent bottom density currents from a high-order nonhydrostatic spectral element model. J Phys Oceanogr 2004;34:2006-26.

[15] Rottman J, Simpson J. Density currents produced by instantaneous releases of a heavy fluid in a rectangular channel. J Fluid Mech 1983;135:95-110.

[16] Stoker JJ. Water waves. New York: Interscience; 1957. pp. 333-40.

[17] Ungarish M, Zemach T. On the slumping of high Reynolds number gravity currents in two-dimensional and axisymmetric configurations. Eur J Mech B/ Fluids $2005 ; 24: 71-90$

[18] Ungarish M. A shallow-water model for high-Reynolds-number gravity currents for a wide range of density differences and fractional depths. J Fluid Mech 2007;579:373-82.

[19] Ungarish M. An introduction to gravity current intrusions. CRC Press; 2009.

[20] Zalesak ST. Fully multidimensional flux-corrected transport algorithms for fluids. J Comput Phys 1979;31:335-62. 